\title{
Good practice for radiological reporting. Guidelines from the European Society of Radiology (ESR)
}

\author{
European Society of Radiology (ESR)
}

Received: 3 January 2011 / Accepted: 3 January 2011 / Published online: 6 February 2011

(C) European Society of Radiology 2011

\begin{abstract}
The views of the European Society of Radiology concerning what constitutes a good radiological report are outlined in this article. Some pertinent literature is reviewed.
\end{abstract}

Keywords Radiology report · Quality

\begin{abstract}
Aim of the document
There is no universally agreed definition of a good radiological report, and both radiologists and those who receive reports may hold differing views on the optimal style and content. Nevertheless, all radiologists wish to produce reports which are accurate and also interpret the investigation for a particular patient in a way that assists with their further management.

Medicine is an international subject and, with the advent of picture archiving and communication systems (PACS), images may be transported throughout Europe and indeed the world. Considerable variation currently exists in the way imaging reports are constructed. It can be argued that there is benefit in reducing this variation for ease of comprehension on the part of referrers, and to aid audit, teaching and research.

This document suggests a framework, which it is hoped will be relevant to radiological practice throughout Europe.
\end{abstract}

European Society of Radiology (ESR)

Neutorgasse $9 / 2$,

1010 Vienna, Australia

e-mail: communications@myESR.org

URL: www.myESR.org

\section{Introduction}

The written radiological report is the most important means of communication between the radiologist and referring medical doctor [1-3]. It is part of the patient's permanent health record, and interprets the investigation in the clinical context. The appropriate construction, clarity, and clinical focus of a radiological report are essential to high quality patient care [4].

\section{Knowledge, skills and training required [2]}

Knowledge

1. Sufficient medical and surgical knowledge to interpret the clinical information provided and understand the suspected underlying pathology

2. Sufficient knowledge to judge the appropriateness of the examination relative to the clinical information provided and other methods of investigation available

3. In-depth knowledge of the accuracy, reliability and potential complications of the investigation

4. Familiarity with the radiological abnormalities shown and with their significance

5. Appreciation of normal variants which may mimic disease

6. An understanding of the relationship between the imaging appearance and the underlying pathology

7. Knowledge of when urgent action is required on the basis of the imaging findings

\section{Skills [5]}

1. Justification: the ability to make judgements on the clinical justification for the examination 
2. Optimisation: the ability to carry out or protocol and optimise the examination

3. Technical: the ability to maximise image and contrast resolution, to minimise exposure to ionizing radiation and to perform relevant image manipulation

4. Observational: the ability to identify radiological abnormalities and distinguish them from normal appearances and variants

5. Image analysis: the ability to evaluate abnormal imaging features and relate them to underlying causative pathology

6. Communication: the ability to convey the interpretation of the investigation clearly, both verbally and in writing, and to communicate directly with the patient where appropriate

\section{Training}

1. Sufficient training to satisfy local and national regulatory requirements for image interpretation

2. Appropriate training to perform and interpret the particular imaging investigation undertaken

\section{Elements of a report}

Although there are no universally accepted rules for the structure of a radiological report, it can be argued that concise, consistent ordering of the report both reduces variation between reports and makes it easier for referrers who become familiar with the format to assimilate the information $[1,4,6]$

The broad categories can be summarised as:

- Clinical referral

- Technique

- Findings

- Conclusion

- Advice

\section{Clinical referral}

This section should include a brief summary of the reason for referral, summarising the clinical problem. It should be as concise as possible, but should nevertheless contain all the relevant clinical information. It is of the utmost importance that the clinical history of the patient is provided by the referring physician, in order to enable correct image interpretation. If insufficient clinical information is available for the radiologist to give a full interpretation, this should be stated in the report. When appropriate, the justification of radiation exposure should be given.
Technique

This section should include:

(a) A concise description of the investigation/procedure performed, with specific mention of any non-standard elements of the investigation, e.g. additional sequences.

(b) A record of contrast medium administration, including route of administration, type and dose. Any adverse reaction must be recorded and the treatment described. All additional medications that were administered to the patient while in the imaging department (e.g. sedatives) should also be recorded.

(c) A description of technically suboptimal features if they may have an impact on the accuracy of interpretation.

(d) Patient radiation dose where applicable.

Findings

(a) This section of the report should include a targeted, systematic and comprehensive description of all abnormalities and should start with those features that are relevant to the clinical request or suspected pathology.

(b) Observations should be described using accepted imaging terminology, and should be as precise as possible, avoiding loose terms such as 'shadowing'.

(c) The description should be specific in giving the dimensions, signal intensity, attenuation, echogenicity or density of abnormalities. Specific positive or negative features which will affect interpretation of the abnormality/-ies, such as clarity of margin, calcification or cavitation should also be described.

(d) The anatomical site of abnormalities should be clearly stated, together with their relationship to other structures where appropriate.

(e) It may be helpful to the referrer and anyone subsequently viewing the images to indicate the relevant images on which the abnormality is best shown and on which the measurements were performed, e.g. series 2, image 12 .

(f) Relevant negative findings should be specifically stated.

(g) Incidental findings should be stated and analysed.

(h) Where previous investigations have been performed, comparison with the current examination should be carried out and described in the report, including the date of the previous examination. The absence of previous imaging should also be recorded if relevant.

Impression/conclusion

(a) This comprises an interpretation of the investigation, taking into account all the imaging features, together 
with relevant clinical information and laboratory findings, to formulate an overall impression.

(b) The aim is to reach a precise diagnosis when possible, or an appropriately ranked differential diagnosis.

(c) Where a differential diagnosis is given, it should be relevant and limited, and the evidence supportive of or against each suggested diagnosis should be explained.

(d) The conclusion should relate to the original presentation, e.g. 'no cause for the left chest pain identified'.

(e) Any incidental findings should be clearly described as either relevant or non-significant.

(f) Any adverse event should be restated.

\section{Advice}

The report may give suggestions for further action to be taken, e.g. referral for an urgent specialist opinion. More commonly, advice will be given on further investigations which will refine the diagnosis. These suggestions should be carefully considered and not expose the patient to unnecessary further investigations.

\section{Validation of report}

All imaging reports must be checked and signed with electronic or other signature.

\section{Length of report}

A balance needs be struck between a clear description of the positive and negative findings and the concentration of the reader. There is evidence that a large number of referrers do not read the whole report [3]. The impression or conclusion section is therefore critical, and it should be assumed that in some cases it is all that will be read.

\section{Language used}

The wording of the report should take into account the expected level of knowledge and expertise of the referrer. A specialist may be more familiar with certain medical abbreviations than a referrer outside that particular specialist area. Long descriptions of limited interest to the referrer should be avoided. Abbreviations should be used only when it is clear that the referrer will be familiar with the relevant terminology.

\section{Style and structure}

Traditionally, radiologists have developed their own personal style of reporting, which may be tailored to the type of work they carry out, the general style in use within their department/ institution, or the demands of local referrers. Many radiologists will, over time, refine their style in a way which will become very familiar to their key clinical referrers.

\section{Structured reporting: the future?}

Conventional radiology reports are stored as free text, so information is trapped in the language of the report, making it difficult to find specific details without reading the whole text. In structured reporting (SR), the information is standardised and presented in a clear, organised format, tracking the attributes of each finding (size, location, etc.) and prompting the radiologist to complete all required fields. It has been suggested that SR is more time-efficient than dictation, facilitates automated billing and order entry, and supports analysis for research and decision-support [7]. SR has also been suggested to improve communication of radiology results $[4,8]$ and allows retrieval of data by automated or semi-automated methods for the purposes of comparison, audit and research.

SR is usually displayed in modular format with section headings, contains a consistent ordering of observations in the form of templates or checklists, and uses standardised language and lexicon [4, 9-11]. There is also the potential to integrate additional information, such as clinical data, technical parameters, measurements, annotations, and key (relevant) images and multimedia data [10], giving the potential to reduce ambiguity and increase confidence in the findings $[12,13]$. There is also the future potential for multilingual translation.

Technical frameworks for SR are potentially complex and the ESR will undertake to provide further guidance in future documents.

\section{Emergency or unexpected findings}

If the examination reveals an abnormality which requires urgent further evaluation or treatment, the radiologist has a duty of care to communicate this to the referring medical doctor. The date, time and person notified and the means of notification should be clearly stated in the report (ideally at the beginning or end of the report, rather than within the body of the report).

The situation may be encountered which is not an emergency, but where there may be adverse consequences for the patient if a significant unexpected finding revealed 
on imaging is not subsequently acted upon. Here the duty is less clear, but 'alert' methods for referrers should be in place so that such patients are further investigated or treated in an appropriate time frame [14].

\section{Summary}

In a European setting of cross-border healthcare, there are benefits in moving towards a more uniform style and structure of radiological reports. This would provide a more consistent service to patients and referrers. It would also facilitate audit, teaching and research.

Acknowledgements This article was kindly prepared by the ESR Subcommittee on Audit \& Standards (Chairman: E. Jane Adam. National Societies Committee Chairman: Guy Frija. Members: Hudaver Alper, Éamann Breatnach, Maurizio Centonze, Elisabeth Dion, Birgit Ertl-Wagner, Robert Manns) on behalf of the ESR. It was approved by the ESR Executive Council in October 2010.

\section{References}

1. American College of Radiology (2005) ACR practice guideline for communication of diagnostic imaging findings. American College of Radiology, Reston. Available at www.acr.org Accessed September 2010.
2. The Royal College of Radiologists (2006) Standards for the Reporting and Interpretation of Imaging Investigations. The Royal College of Radiologists, London. Available at www.rcr.ac.uk Accessed September 2010.

3. Societe Français Radiologie, Groupe de travail SFR - CRR (2007) Recommandations générales pour l'élaboration d'un compte rendu radiologique (CRR). J Radiol 88:304-306

4. Kahn CE Jr, Langlotz CP, Burnside ES et al (2009) Towards best practices in radiology reporting. Radiology 252:852-856

5. The Royal College of Radiologists (1999) Skills mix in clinical radiology BFCR(99)3. The Royal College of Radiologists, London. Available at www.rcr.ac.uk Accessed September 2010.

6. Kushner DC, Lucey LL (2005) American College of Radiology. Diagnostic radiology reporting and communication: the ACR guideline. J Am Coll Radiol 2(1):15-21

7. www.structuredreporting.com Accessed September 2010.

8. Dunnick NR, Langlotz CP (2008) The radiology report of the future: a summary of the 2007 Intersociety Conference. J Am Coll Radiol 5(5):626-629

9. Sistrom CL, Langlotz CP (2005) A framework for improving radiology reporting. J Am Coll Radiol 2(2):159-167

10. Reiner BI, Knight N, Siegel EL (2007) Radiology reporting, past, present, and future: the radiologist's perspective. J Am Coll Radiol 4(5):313-319

11. Weiss DL, Langlotz CP (2008) Structured reporting: patient care enhancement or productivity nightmare? Radiology 249:739-747

12. Iyer VR, Hahn PF, Blaszkowsky LS et al (2010) Added value of selected images embedded into radiology reports to referring clinicians. J Am Coll Radiol 7:205-210

13. Reiner B, Siegel E (2006) Radiology reporting: returning to our image-centric roots. AJR Am J Roentgenol 187:1151-1155

14. Boland GW, Guimaraes AS, Mueller PR (2008) Radiology report turnaround: expectations and solutions. Eur Radiol 18(7):13261328 